\title{
Limits of Three-Dimensional Resolution and Dose for Aberration-Corrected Electron Tomography
}

\author{
Reed Yalisove, ${ }^{1, \dagger}$ Suk Hyun Sung $\odot,{ }^{1, \dagger}$ Peter Ercius, ${ }^{2}$ and Robert Hovden ${ }^{1,3, *}$ \\ ${ }^{1}$ Department of Materials Science and Engineering, University of Michigan, Ann Arbor, Michigan 48109, USA \\ ${ }^{2}$ The Molecular Foundry, Lawrence Berkeley National Laboratory, Berkeley, California 94720, USA \\ ${ }^{3}$ Applied Physics Program, University of Michigan, Ann Arbor, Michigan 48109, USA
}

(Received 28 July 2020; revised 28 September 2020; accepted 2 December 2020; published 5 January 2021)

\begin{abstract}
Aberration-corrected electron microscopy can resolve the smallest atomic bond lengths in nature. However, the high-convergence angles that enable spectacular resolution in two dimensions have unknown three-dimensional (3D) resolution limits for all but the smallest objects ( $<\sim 8 \mathrm{~nm}$ ). We show aberrationcorrected electron tomography offers new limits for 3D imaging by measuring several focal planes at each specimen tilt. We present a theoretical foundation for aberration-corrected electron tomography by establishing analytic descriptions for resolution, sampling, object size, and dose - with direct analogy to the Crowther-Klug criterion. Remarkably, aberration-corrected scanning transmission electron tomography can measure complete 3D specimen structure of unbounded object sizes up to a specified cutoff resolution. This breaks the established Crowther limit when tilt increments are twice the convergence angle or smaller. Unprecedented 3D resolution is achievable across large objects. Atomic 3D imaging ( $1 \AA$ ) is allowed across extended objects larger than depth of focus (e.g., $>20 \mathrm{~nm}$ ) using available microscopes and modest specimen tilting $\left(<3^{\circ}\right)$. Furthermore, aberration-corrected tomography follows the rule of dose fractionation where a specified total dose can be divided among tilts and defoci.
\end{abstract}

DOI: 10.1103/PhysRevApplied.15.014003

\section{INTRODUCTION}

Among the greatest goals in experimental science is to directly measure the complete three-dimensional (3D) arrangement of atoms in materials. However, fundamental sampling limits and an inextricable connection between lateral resolution and depth of focus have strictly prohibited $3 \mathrm{D}$ atomic measurement of extended materials. The theoretical limits of electron tomography have long been defined by (1) the Crowther-Klug criterion, which relates $3 \mathrm{D}$ resolution to the number of projections acquired [1], and (2) the dose-fractionation theorem [2], which states that the significance of a reconstructed object is independent of the distribution of dose. These limits were developed on the assumption that each image in a tomographic tilt series gives a perfect projection of the specimen. Over the last half century, this assumption has sufficed for microscopes where the depth of focus is large relative to the specimen [3-7].

\footnotetext{
*hovden@umich.edu

$\dagger$ These authors contributed equally to this work.

Published by the American Physical Society under the terms of the Creative Commons Attribution 4.0 International license. Further distribution of this work must maintain attribution to the author(s) and the published article's title, journal citation, and DOI.
}

However, the defined limits of tomography are invalid for the new era of aberration-corrected scanning transmission electron microscopy (STEM) where highly convergent electron beams confined to sub-Ångstrom lateral dimensions [8] provide routine atomic imaging in two dimensions [9-11]. These revolutionary microscopes no longer provide simple projections of a specimen [12-15] and tomography fails for objects larger than the depth of focus $(<\sim 8 \mathrm{~nm})$. Hovden et al. experimentally showed overcoming the limitations of aberration-corrected STEM tomography requires collecting a through-focal image stack at every specimen tilt [16]. Although experimentally demonstrated $[16,17]$, the theoretical limits of aberrationcorrected tomography remain undefined. Understanding the trade-off between resolution, object size, sampling, and dose using highly convergent beams demands a new theoretical definition.

Here we present a theoretical foundation for aberrationcorrected electron tomography that establishes analytic descriptions for resolution, sampling, and object size. We show that aberration-corrected tomography can far exceed the resolution limits of traditional tomography and breaks the conventional Crowther-Klug criteria.

The 3D contrast-transfer-function (CTF) for aberrationcorrected STEM tomography distinctly measures a volume of information made from a superposition of toroids with petal-shaped cross sections (Fig. 1) at every specimen tilt. 


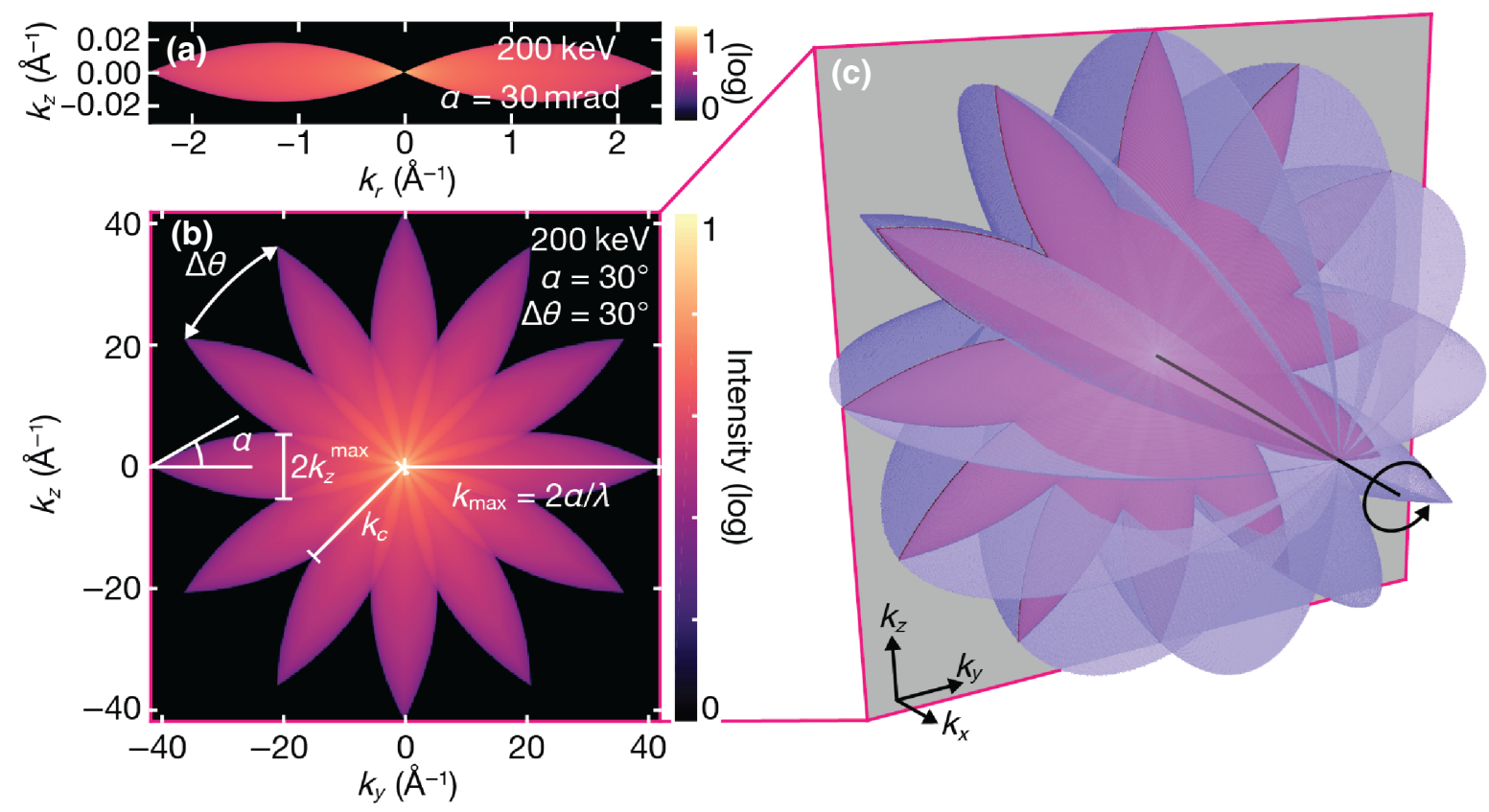

FIG. 1. 3D contrast-transfer-function (CTF) tomography using aberration-corrected scanning transmission electron microscopy. (a) Through-focal CTF for typical aberration-corrected STEM ( $\alpha=30 \mathrm{mrad}, 200 \mathrm{keV})$. (b) Internal structure of the tomographic CTF at $k_{x}=0$ is highlighted. Each petal-shaped lobe represents a single through-focal CTF. Complete transfer of information is guaranteed within a spherical radius $k_{c}$. (c) 3D CTF of through-focal tomography (tilt axis, $\hat{\mathbf{x}}$ ). Blue shell denotes the information transfer limit in three dimensions. $\alpha$ and $\Delta \theta$ are exaggerated to $30^{\circ}$. The plane slices through the CTF at $k_{x}=0$.

A remarkable feature of the $3 \mathrm{D}$ CTF is that the overlapped regions permit complete $3 \mathrm{D}$ information collection up to a specified resolution $\left(1 / k_{c}\right)$ - unachievable with conventional tomography. This occurs when the incremental tilt angle $(\Delta \theta)$ becomes smaller than twice the beam convergance semiangle $(2 \alpha)$, which is typical to instrument operation $\left(\alpha>25 \mathrm{mrad}\right.$, approximately $\left.1.4^{\circ}\right)$ and sampling $\left(\Delta \theta<2^{\circ}\right.$, approximately $\left.35 \mathrm{mrad}\right)$. This complete information transfer breaks the Crowther-Klug relationships and the maximum reconstructable object size is unlimited up to a critical resolution $\left(1 / k_{c}\right)$. Beyond this critical resolution, Crowther-like trade-offs define the maximum object size $(D)$ allowed at a given $3 \mathrm{D}$ resolution $(d)$. With more specimen tilts and higher convergence angles, 3D resolution improves quickly and the maximum object size increases dramatically. Using a tilt increment that matches the convergence angle $(\Delta \theta=\alpha)$ any object size can be resolved with $3 \mathrm{D}$ resolution at approximately $50 \%$ of the microscope's diffraction limited resolution.

Despite the large amount of image data required by aberration-corrected electron tomography, the dose can be chosen to mitigate total specimen exposure. Extending the dose-fractionation arguments presented by Hoppe [2] and Saxton [18], we show aberration-corrected electron tomography allows tunable dose allocation across any number of tilts and focal planes when oversampled. Thus, the signalto-noise ratio of a $3 \mathrm{D}$ reconstruction is only dependent on the total dose imparted.
The relationships defined herein for aberration-corrected tomography supplant the Crowther-Klug criterion [1,19] and the dose-fractionation theorem $[2,18]$ that have long defined traditional tomographic techniques. Moreover, this work extends beyond scanning transmission electron tomography and is applicable to any incoherent linear imaging technique that uses highly convergent beams where the depth of focus is small compared to the $3 \mathrm{D}$ object size.

\section{BACKGROUND}

In 1970, Crowther et al. established the fundamental trade-off between 3D resolution, specimen size, and the number of projections measured [19]. Bracewell and Riddle showed the same relationship for radio astronomy in 1967 [20]. With evenly spaced specimen tilts about a single axis of rotation, the expression is compactly stated: $d=\pi D / N$, where $d$ is the smallest resolved feature size in three dimensions, $D$ is object size, and $N$ is the number of projections acquired with equal angular spacing $(\Delta \theta=\pi / N)$. This sampling criterion is the most stringent requirement that can be adopted and ensures specimen features are measured and the entire reconstruction is equally sharp and free of aliasing.

Conceptually, 3D resolution is limited by tomography's inability to collect complete information about the specimen. Projection images at each tilt map to a plane of 
information in frequency space ( $k$ space) - as defined by the projection slice theorem [21]. The missing information between planes limits the 3D resolution and object size of a tomographic reconstruction. For specimens tilted about a single axis of rotation, the planes of information intersect along one axis $\left(k_{x}\right)$ on a cylindrical coordinate system as illustrated in Fig. SI3 within the Supplemental Material [22]. Sampling is maximal radially and along the axis of rotation, however undersampling occurs azimuthally along $k_{\theta}$ and worsens at higher frequencies $\left(k_{r}\right)$. The azimuthal gap between adjacent measurement planes $\left(\Delta k_{\theta} \approx k_{r} \times\right.$ $\Delta \theta)$ limits the largest resolvable object: $\Delta k_{\theta}=1 / D$. Thus, collecting more specimen tilts reduces the distance between measurement planes and allows higher resolution (larger $k_{r}$ ) or larger object sizes in three dimensions. This theorem is well suited for traditional (S)TEM tomography where the depth of focus is larger than the object size (Fig. SI2 within the Supplemental Material [22]).

Six years after Crowther et al. defined the resolution and sampling limits of tomography, Hegerl and Hoppe established a dose-fractionation property for tomography, which Saxberg and Saxton further refined after debate $[2,18]$. Their work showed when an object is sufficiently sampled (i.e., better than Crowther-Klug requirements) the SNR of a reconstruction depends only on the total dose imparted. Maintaining an equivalent total dose, one may divide that dose across more or fewer projections. Both derivations are based on weak-contrast imaging - however, we show a weak-contrast approximation is not required.

\section{CONTRAST TRANSFER FUNCTION OF ABERRATION-CORRECTED TOMOGRAPHY}

Due to the highly convergent nature of aberrationcorrected electron beams, Crowther's derivation of sampling requirements do not hold for aberration-corrected tomography. The CTF is no longer a two-dimensional plane; its form becomes a 3D toroid [Fig. 1(a)] derived analytically by Intaraprasonk et al. [23] and reproduced in Fig. SI1 within the Supplemental Material [22]. Thus for aberration-corrected tomography, the projection planes in $k$ space are replaced by toroidal CTFs. Figures 1(b) and 1(c) show the total tomographic CTF in three dimensions. Summing a rotated set of toroidal CTFs describes the region of $k$ space from which information is collected in aberration-corrected tomography:

$$
H\left(k_{r}, k_{z}\right)=\sum_{\theta} h\left(k_{r}^{\theta}, k_{z}^{\theta}\right) .
$$

Here $h\left(k_{r}^{\theta}, k_{z}{ }^{\theta}\right)$ is the radially symmetric toroidal CTF from a single specimen tilt, $\theta$, measured by through-focal imaging. For an aberration-free beam this through-focal
$\mathrm{CTF}$ is

$$
\begin{gathered}
h\left(k_{r}^{\theta}, k_{z}^{\theta}\right)=\frac{1}{2 \pi^{3} \alpha k_{r}^{\theta}} \sqrt{1-\left(\frac{k_{r}^{\theta} \lambda}{2 \alpha}+\frac{\left|k_{z}^{\theta}\right|}{\alpha k_{r}{ }^{\theta}}\right)^{2}} \\
\left|k_{z}^{\theta}\right| \leq \frac{\lambda}{2} k_{r}^{\theta}\left(\frac{2 \alpha}{\lambda}-k_{r}^{\theta}\right),
\end{gathered}
$$

where $\alpha$ is the convergence semiangle of the electron beam, $k_{r}$ is the radial frequency, and $\lambda$ is the wavelength of the electron. By parameterizing the equation for the through-focal CTF [Eq. (2)], we express a CTF that has been tilted about an axis $\left(k_{x}\right)$ perpendicular to the optical axis $\left(k_{z}\right)$ in $k$ space. This rotation gives $k_{r}^{\theta}=$ $k_{r} \cos (\theta)-k_{z} \sin (\theta)$ and $k_{z}{ }^{\theta}=k_{r} \sin (\theta)+k_{z} \cos (\theta)$ for $\theta \in(-\pi / 2, \pi / 2]$. Each CTF is bounded by Eq. (3). These equations describe an aberration-free (chromatic and geometric) probe formed by an infinitesimal source within an objective aperture that limits aberrations and sets the convergence semiangle (Fig. SI1 within the Supplemental Material [22]). This aberration-corrected tomography CTF is valid under a linear incoherent imaging approximation.

The CTF for aberration-corrected tomography [Eqs. (1)(3)] is shown in Figs. 2(a) and 2(b) for a 200-keV electron beam with $30-\mathrm{mrad}$ convergence semiangle and a $2^{\circ}$ tilt interval about a single axis of rotation.

\section{3D RESOLUTION AND OBJECT SIZE LIMITS}

For aberration-corrected tomography, the sampling requirements that limit object size and resolution are determined by the missing information between the toroidal bounds of the through-focal CTF [Eq. (3)]. For infinitely large objects, measuring specimen structure at a single frequency $\left(\mathbf{k}^{\prime}\right)$ requires the information to lie within the tomography CTF. However, for objects of finite size, $D$, the condition loosens and CTF bounds only need to measure the neighborhood, $\mathbf{k}^{\prime}+\boldsymbol{\delta}$ where $|2 \boldsymbol{\delta}|=1 / D$ (Fig. 5 within the appendix). Thus, the size of missing information in $k$ space limits the detectable object size. Similar to the analysis by Crowther and Klug, we aim to calculate the maximum object size for a tomographic reconstruction by calculating the $k$-space distance, $\Delta k_{\theta}$, between the information collected at sequential specimen tilts. The nonplanar geometry of aberration-corrected tomography reduces distances of missing information in $k$ space that permit measurement of larger real-space objects at higher resolutions than the Crowther-Klug limit.

The strictest resolution requirement ensures measurement of objects of any shape or symmetry without any prior information. Although the tomographic CTF is nonisotropic and resolution is higher along the axis of rotation, we define 3D resolution by the worst measurable resolution. For single tilt-axis tomography, undersampling 

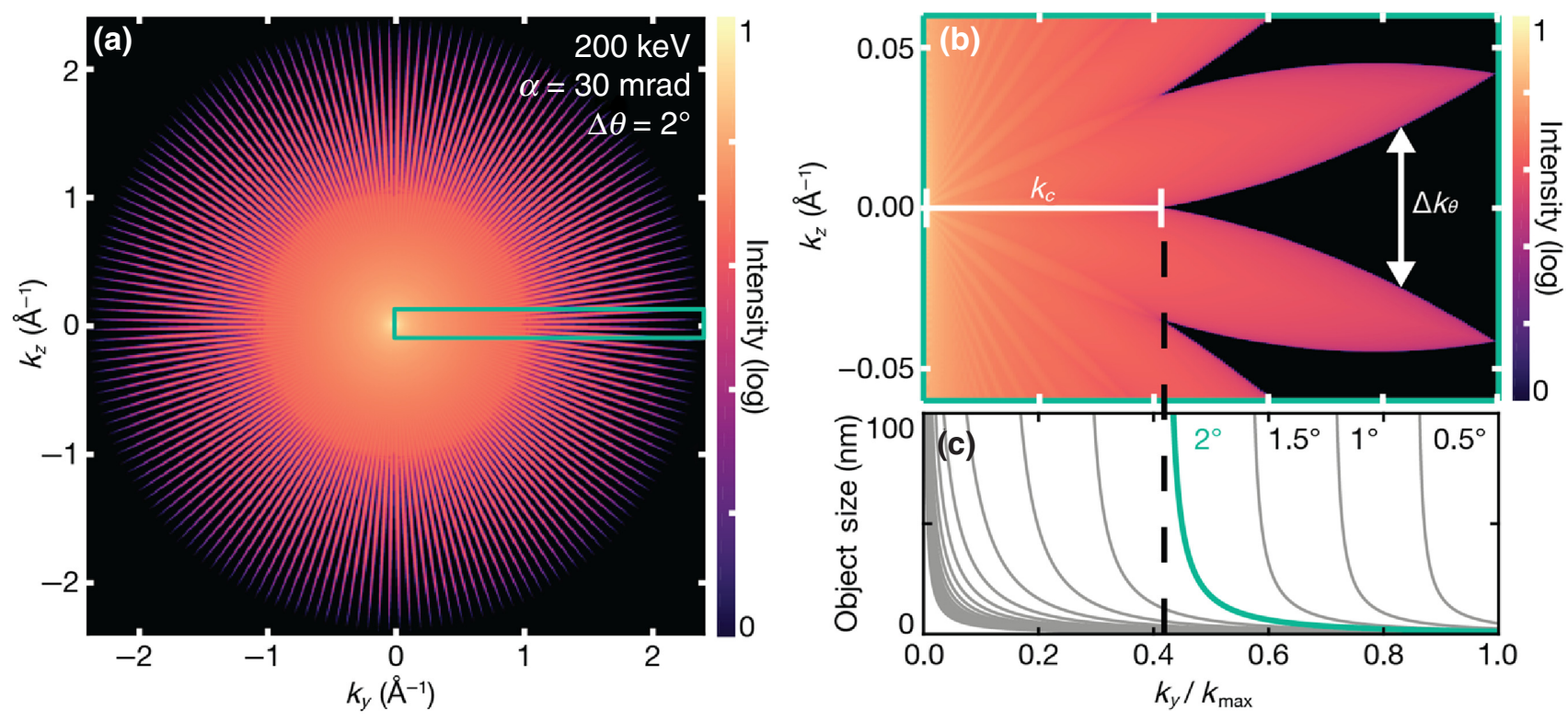

FIG. 2. Relationships between tilt angle, resolution, and maximum object size. The CTF of aberration-corrected tomography hosts overlap regions, which permit complete information transfer - and therefore unlimited object size with resolution $d=1 / k_{c}$. (a) Full tomographic CTF in cross section for $200 \mathrm{keV}, 30 \mathrm{mrad}, 2^{\circ}$ tilts. (b) Subregion of full tomographic CTF highlights the maximum frequency of complete information transfer, $k_{c}$, and $\Delta k_{\theta}$ denotes separation between through-focal CTFs at each tilt. (c) Spatial frequency versus maximum object size for several tilt step sizes with the teal curve matching conditions in (a). For $k_{y}<k_{c}$, the maximum object size is unbounded.

occurs along $k_{\theta}$ and defines the largest $k$-space distance between adjacent through-focal CTFs. The missing information $\left(\Delta k_{\theta}\right)$ increases at higher frequencies, $k_{r}$, and thus limits resolution $\left(d=1 / k_{r}\right)$. We show (Appendix A) the distance between measured information is maximal on the $k_{y}-k_{z}$ plane (Fig. 1) and is used to calculate the limits for aberration-corrected tomography.

The most striking feature of the aberration-corrected tomography CTF is the continuum of information it can permit. From Fig. 1(b) we see that with small tilt increments and large convergence angles, adjacent throughfocal CTFs will overlap, allowing complete information transfer up to a critical frequency, $k_{c}$ :

$$
k_{c}=\frac{2 \alpha-\Delta \theta}{\lambda},
$$

where $\Delta \theta$ is the angular spacing between specimen tilts. Equation (4) defines this critical frequency under a firstorder small-angle approximation as derived in Appendix A and is valid when $k_{c}$ is positive (i.e., $\Delta \theta<2 \alpha$ ).

This critical frequency splits the problem into two regimes, so the resolution limit on object size is defined piecewise. For $k_{r} \leq k_{c}$, the structure of the specimen is completely measured - this corresponds to unbounded maximum object sizes. For $k_{r}>k_{c}$, there is a finite distance between adjacent regions of information [Eq. (A2) within the appendix], which relates the maximum frequency, $k_{y}$, in a reconstruction to the maximum object size,
$D$ [shown in Fig. 2(c)]. The piecewise expression is

$$
D= \begin{cases}\frac{d^{2}}{\lambda\left(1-k_{c} d\right)}, & \frac{\lambda}{\alpha}<d<\frac{1}{k_{c}} \\ \infty, & \frac{1}{k_{c}} \leq d<\infty\end{cases}
$$

Equation (5) defines a limit relating resolution, object size, and sampling for aberration-corrected electron tomography, analogous to the Crowther-Klug limit for conventional tomography. It shows higher beam energies (i.e., smaller wavelengths) and higher convergence angles allow higher resolution and larger object sizes.

Remarkably, when $\Delta \theta<2 \alpha$, there is always a resolution at which an infinite object size can be reconstructed (neglecting multiple scattering). This behavior is not predicted by the Crowther criterion and exceeds the previously expected limits. As illustrated in Fig. 3, with even smaller tilt increments the reconstructable object size diverges at high resolution. Notably, when the tilt increment matches the convergence angle $(\Delta \theta=\alpha)$ any object size can be resolved in three dimensions at approximately $50 \%$ of the microscope's diffraction limit. For $\Delta \theta=\alpha / 2 \mathrm{a}$ $3 \mathrm{D}$ resolution at $75 \%$ the diffraction limit is achievable for an unlimited object size.

At higher resolution beyond the critical frequency, $k_{c}$, aberration-corrected tomography still outperforms traditional tomography due to the reduced missing information between lobes in the tomography CTF. Figure 3(b) shows 


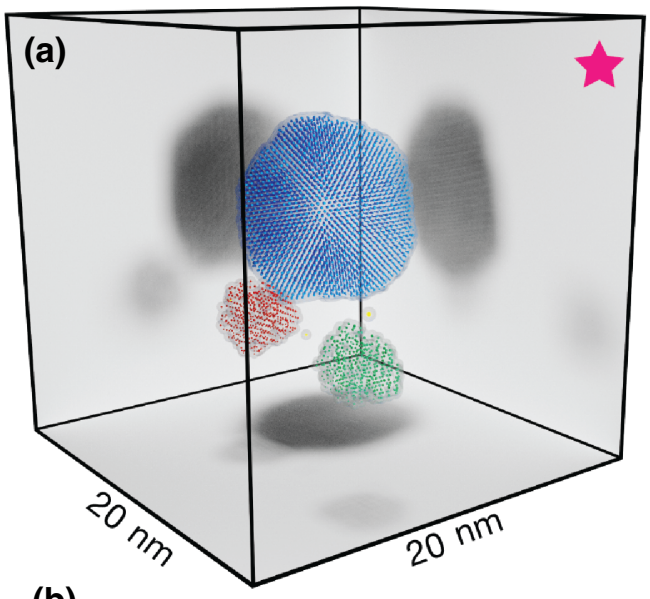

(b)

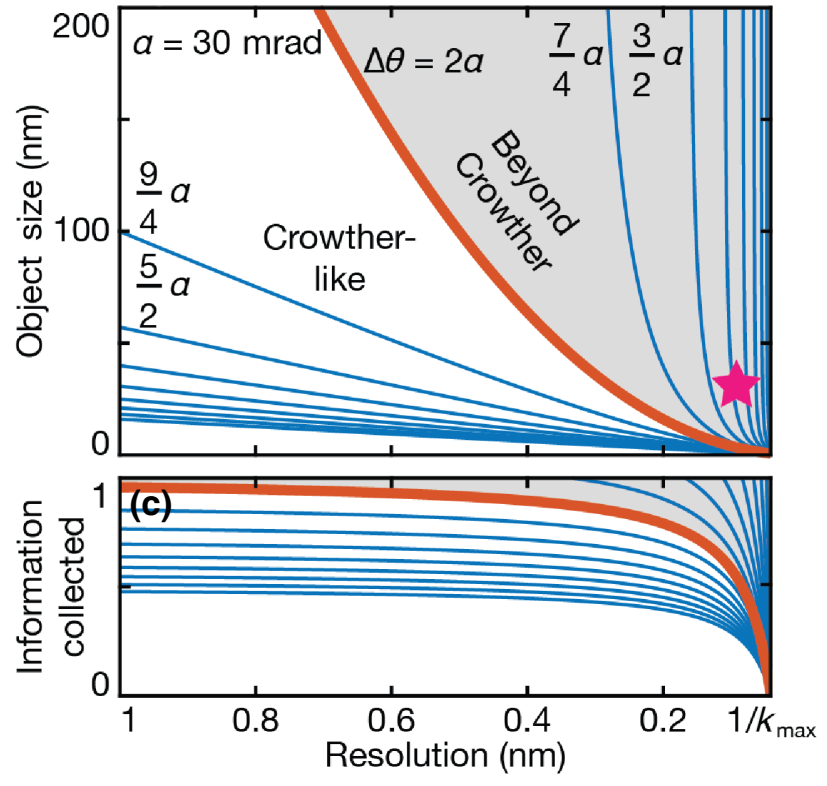

FIG. 3. Aberration-corrected electron tomography enables unprecedented high resolution of extended objects. (a) 3D atomic resolution tomography of three nanoparticles in a $20 \mathrm{~nm}$ volume - reconstructed here from quantum-mechanical scattering simulations. (b) Trade-off between 3D resolution and object size is plotted for different specimen tilt increments, $\Delta \theta$. The Crowther limit is surpassed when $\Delta \theta \leq 2 \alpha$ (gray) such that objects of any size may be reconstructed. Star denotes the size and resolution of reconstruction in (a). (c) The percent of information collected at each resolution.

the trade-off between object size and resolution is favorably nonlinear. It provides the object size that can be reconstructed at a given resolution for different specimen tilt increments. For example, a 75-nm specimen, imaged with a 30 -mrad convergence angle and $3^{\circ}(50-\mathrm{mrad})$ increments between tilts allows $2-\AA$ resolution in three dimensions at $200 \mathrm{keV}$.

Moreover, atomic resolution imaging, with $1.5-\AA$ resolution in $3 \mathrm{D}$, is possible over a $15-\mathrm{nm}$ object if sampled at $3^{\circ}$ using a $200-\mathrm{keV}$ beam and $30-\mathrm{mrad}$ convergence semiangle. $3 \mathrm{D}$ atomic resolution imaging of extended objects is computationally verified [Fig. 3(a)] using quantum-mechanical multiple-scattering simulations (i.e., multislice) of aberration-corrected tomography performed on crystalline nanoparticles within a $20-\mathrm{nm}$ volume (see Fig. SI2 within the Supplemental Material [22]). This simulation computed over 500 million elastically scattered electron wave functions to generate images at 13 defocus positions at each of 105 tilts, using over 15000 -GPU core hours. Computed through-focal stacks are interpolated onto a complex Cartesian grid in Fourier space. The final reconstruction is obtained directly from the 3D inverse Fourier transform (see Fig. SI2 within the Supplemental Material for further details [22]).

Despite some missing information at higher frequencies $\left(k_{r}>k_{c}\right)$, a significant portion of $k$ space is measured and aberration-corrected tomography provides a superior reconstruction compared to traditional tomography. However, due to the finite periodic sampling of specimen tilts, aberration-corrected tomography may still permit weak aliasing. Aliasing can occur azimuthally at high radial frequencies, $k_{r}>k_{c}$, when reconstructed object sizes exceed the Crowther-Klug relation, even if the requirements for resolution [Eq. (5)] are met. Fortunately, if present, aliasing is substantially attenuated by the amount of information collected with aberration-corrected tomography. The intensity of azimuthal aliasing is proportional to the percentage of information collected at each radial frequency $k_{r}$ [plotted as a function of resolution in Fig. 3(c)]. With more measured information, the reconstruction quality improves and aliasing becomes negligible. Thus, no aliasing occurs at low frequencies $\left(k_{r}<k_{c}\right)$ and at the microscope's transfer limit $\left(k_{r}=k_{\max }\right)$ the aliasing is significant and matches traditional tomography.

\section{DEFOCUS SAMPLING REQUIREMENT}

At each specimen tilt, aberration-corrected electron tomography acquires a through-focal stack of images. This overcomes the limited depth of focus $(<\sim 8 \mathrm{~nm})$ where a single image cannot sufficiently measure large objects. With through-focal imaging, the focal plane incrementally moves through the entire object and measures specimen information within a through-focal CTF [Eq. (2)]. The defocus step size, $\Delta z$, becomes an additional sampling requirement.

The defocus step must be smaller than the microscope's depth of focus. This sampling requirement is described by the widest portion of a through-focal CTF $\left(k_{z}^{\max }\right)$ along the beam direction $\left(k_{z}\right)$. The largest defocus step size is

$$
\Delta z^{\max }=\frac{1}{2 k_{z}^{\max }}=\frac{\lambda}{\alpha^{2}} .
$$


This equation is the well-known depth-of-focus relationship, and is analytically derived via wave optics in Appendix B.

Ideally, the focal range should not exceed the object being measured as images captured beyond the object bounds increases dose to the sample without adding information. The most dose-efficient measurement has a field of view and defocus range that matches the object size.

\section{DOSE FRACTIONATION}

Surprisingly, aberration-corrected tomography is not necessarily dose intensive. Expanding the dose-fractionation theorem the total dose can be chosen and distributed among both specimen tilts and defoci. Hegerl and Hoppe's original construction for conventional electron tomography states that the SNR of reconstructed voxels depends only on the total dose imparted, not the distribution of dose. It assumes weak-contrast imaging with additive noise $[2,18]$. We use a more complete noise model with Poisson statistics [24]. For a Poisson-limited signal, each noisy image $\tilde{p}(x, y)$ of projected object $p(x, y)$ has a SNR of $\mathcal{R}[\tilde{p}(x, y)]=1+\chi t p(x, y)$ for acquisition time $t$ and dose rate $\chi$ (see Appendix C). For tomography, the signal and noise variance from projections at each tilt add linearly to the final reconstruction because the noise from each image is uncorrelated. Dose fractionation shows that the SNR for projection images - and tomographic reconstructions thereof - depends on both the total dose and the specimen.

$3 \mathrm{D}$ reconstruction quality is independent of the number of specimen tilts only when $k$ space is sufficiently sampled (i.e., oversampled) and the total dose is evenly distributed across equally spaced tilts. The same is true for aberrationcorrected tomography where the signal and noise from volumetric through-focal CTFs add linearly in $k$ space. The SNR of the final reconstruction depends on the total dose imparted onto the specimen not the number of specimen tilts - so long as $k$ space is sufficiently sampled. Notably, oversampling is guaranteed below $k_{c}$ [Eq. (4)].

However, for aberration-corrected tomography, dose is not only divided among tilts, but also among defoci. Here, dose fractionation also holds for throughfocal image acquisitions. SNR of a through-focal stack describes the quality of data at a given dose and dose distribution (see Appendix D). In an oversampled through-focal image stack $\left(\Delta z \ll \Delta z^{\max }\right)$, adjacent defocused images can be summed without loss of information [Figs. 4(a) and 4(b)]. The SNR after sum$\operatorname{ming} M$ adjacent images $\left(\mathcal{R}\left[\sum_{\Delta z} \tilde{p}\left(x, y, z_{f}+\Delta z\right)\right]=1+\right.$ $M \chi t \bar{p}(x, y))$ across defoci $z_{f}+\Delta z$ where dose per image $\chi t$ matches that of a single image, $\bar{p}\left(x, y, z_{f}\right)$, taken with the same total dose $(M \chi t)$. It is the total dose across all images, not the number of images, that determines the (a)

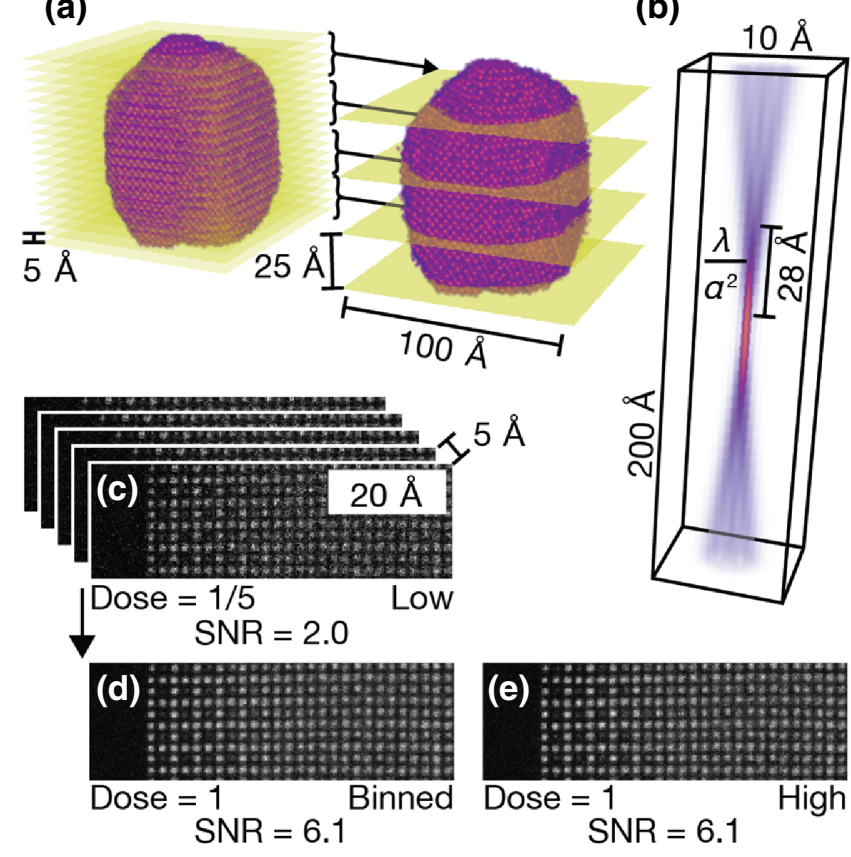

FIG. 4. Dose fractionation for through-focal acquisition (a) Oversampling of focal planes contains the same information as fewer focal planes, so long as the defocus sampling requirement is met. (b) Defocus sampling requirement is set by the depth of focus $\left(\Delta z^{\max }, 28 \AA\right.$ for $\left.200 \mathrm{keV}, 30 \mathrm{mrad}\right)$. Multislice simulation with Poisson noise shows the equivalence of SNR in (c),(d) the sum over five adjacent low-dose defocused images and (e) a single high-dose image.

SNR of useful information so long as the defocus sampling requirement is met [Eq. (6)].

Fully quantum-mechanical multislice simulations with Poisson noise demonstrate the dose-fractionation theorem for through-focal imaging in Fig. 4. A simulated high-dose image [Fig. 4(e)] and a binned through-focal stack with the same total dose [Figs. 4(c) and 4(d)] have comparable SNR and carry the same information. This demonstration on several adjacent defoci can be generalized to the full range of defoci. When the through-focal stack is evenly oversampled along defocus, the reconstruction quality is dependent only on the total dose, not the distribution.

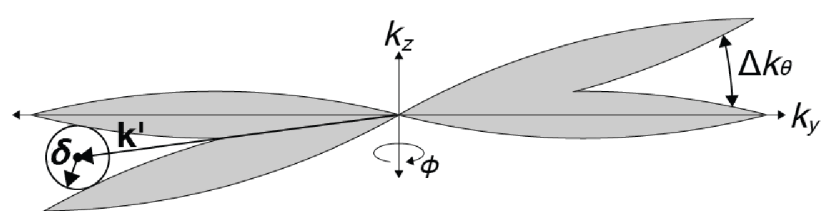

FIG. 5. Illustration of two adjacent tilted CTFs in the plane of maximum separation. On the right, $\Delta k_{\theta}$ separates CTFs, and on the left, the neighborhood about $\mathbf{k}^{\prime}$ with diameter $|2 \boldsymbol{\delta}|=1 / D$ limits the maximum object size. 
Thus, aberration-corrected tomography does not inherently require high doses. The desired total dose for a given specimen determines the SNR of a 3D reconstruction. The total dose may be chosen and divided across tilts and defoci, so long as all sampling requirements are sufficiently met. The traditional dose-fractionation theorem is upheld in aberration-corrected tomography but has the added dimension of defocus sampling. Unfortunately we anticipate aberration-corrected tomography will still adhere to traditional dose requirements where 3D resolution scales inversely with dose ${ }^{1 / 4}[18,25]$ and atomic resolution requires substantial beam exposure.

\section{CONCLUSION}

Aberration-corrected tomography's volumetric CTF breaks the traditional sampling requirements for object size and resolution as famously set by Crowther and Klug [1]. Accounting for the highly convergent imaging probes, a limit on resolution, object size, and sampling is presented in Eqs. (4) and (5). Up to a critical spatial frequency, aberration-corrected tomography can reconstruct an object of any size, and above that frequency the limits on object size still exceed conventional tomography. This is critically significant for the next generation of electron microscopes with ever-increasing convergence angles (>60 mrad) and diminishing depth of focus. Lastly, the signal to noise of a tomographic reconstruction is determined by the total dose of the measurement and that dose may be distributed among defoci and specimen tilt.

With the theoretical limits defined herein, we can proceed to higher resolution across larger fields of view to know the atomic structure of extended specimens in all three dimensions.

\section{ACKNOWLEDGMENTS}

We thank Yi Jiang for helpful discussions. R.Y. and S.H.S. acknowledge support from the DOE BES(j) (Subaward No. K002192-00-S01). R.H. acknowledges support from the Army Research Office, Computing Sciences (W911NF-17-S-0002). Simulations made use of the Advanced Research Computing Technology Services' shared high-performance computing at the University of Michigan and the Molecular Foundry (supported by the Office of Science, U.S. Department of Energy under Contract No. DE-AC02-05CH11231).

\section{APPENDIX A: RESOLUTION, SAMPLING, OBJECT-SIZE RELATIONSHIP FOR ABERRATION-CORRECTED TOMOGRAPHY}

The information measured in reciprocal space by aberration-corrected STEM tomography is described by a superposition of CTFs from each specimen tilt about a single axis of rotation [Eqs. (1)-(3)]. The maximum object size that can be reconstructed with a given resolution is determined by the arc length between adjacent tilted CTFs, $\Delta k_{\theta}$. The upper bound of a CTF in cylindrical coordinates about the $k_{z}$ axis $\left(k_{r}, \phi, k_{z}\right)$ is

$$
k_{z}=\frac{\lambda}{2} k_{r}\left(\frac{2 \alpha}{\lambda}-k_{r}\right)
$$

This upper bound is radially symmetric about the $k_{z}$ axis and the lower bound is a reflection across the $k_{z}=0$ plane. Equivalent points (same $k_{x}$ and $k_{y}$ ) on the bounds of a CTF in Cartesian coordinates are located at $\mathbf{k}_{1}=\left(k_{x}, k_{y}, k_{z}\right)$ and $\mathbf{k}_{2}=\left(k_{x}, k_{y},-k_{z}\right)$.

Fixing $\mathbf{k}_{1}$ and rotating $\mathbf{k}_{2}$ by an angle $\Delta \theta$ about the $k_{x}$ axis we find the vector between adjacent CTFs, $\Delta \mathbf{k}_{\theta}$.

$$
\Delta \mathbf{k}_{\theta}=\left[\begin{array}{c}
0 \\
k_{y}(\cos \Delta \theta-1)+k_{z} \sin \Delta \theta \\
k_{y} \sin \Delta \theta-k_{z}(\cos \Delta \theta+1)
\end{array}\right] .
$$

The magnitude of this vector accurately approximates the arc length between equivalent points on two adjacent CTFs.

$$
\begin{aligned}
\Delta k_{\theta}=\{ & {\left[k_{y}(\cos \Delta \theta-1)+k_{z} \sin \Delta \theta\right]^{2} } \\
& \left.+\left[k_{y} \sin \Delta \theta-k_{z}(\cos \Delta \theta+1)\right]^{2}\right\}^{\frac{1}{2}} \\
= & \sqrt{2}\left[k_{y}{ }^{2}(1-\cos \Delta \theta)\right. \\
& \left.\quad-2 k_{y} k_{z} \sin \Delta \theta+k_{z}^{2}(1+\cos \Delta \theta)\right]^{\frac{1}{2}} \\
= & \sqrt{2}\left(k_{y} \sqrt{1-\cos \Delta \theta}-k_{z} \sqrt{1+\cos \Delta \theta}\right) .
\end{aligned}
$$

Transforming to cylindrical coordinates $\left(k_{y}=k_{r} \sin \phi\right)$, substituting Eq. (A1), and using sine and cosine half-angle identities, the distance between two adjacent CTFs is

$$
\Delta k_{\theta}=2\left[k_{r} \sin \phi \sin \frac{\Delta \theta}{2}+\frac{\lambda}{2} k_{r}\left(k_{r}-\frac{2 \alpha}{\lambda}\right) \cos \frac{\Delta \theta}{2}\right] .
$$

Information is maximally sampled along $k_{r}, k_{x}$ and undersampled along $k_{\theta}$ due to a finite number of specimen tilts. The strictest limit for resolution and object size is set by the path in $k$ space along which $\Delta k_{\theta}$ is largest. We seek to find the plane where $\Delta k_{\theta}$ is maximal and also passes through the origin of $k$ space, so we maximize $\Delta k_{\theta}$ with respect to angle $\phi$ about the $k_{z}$ axis. We need not consider the equivalent angle about the $k_{y}$ axis, as the tomogaphic CTF is symmetric.

$$
\frac{\partial \Delta k_{\theta}}{\partial \phi}=2 k_{r} \cos \phi \sin \frac{\Delta \theta}{2}
$$

Setting $\partial \Delta k_{\theta} / \partial k_{x}=0$, we find an extremum when $\phi=$ $\pi / 2$ (along with trivial extrema $k_{r}=0$ and $\Delta \theta=0$ ). 
The second derivative test shows that $\phi=\pi / 2$ is a maximum, meaning that $\Delta k_{\theta}$ is largest when $k_{r}$ is in the $k_{x}=0$ plane, making the problem two dimensions. The tomography CTF is formed by rotating individual CTFs about the $k_{x}$ axis, so $k_{\rho}$ (a polar coordinate representing distance from the tilt axis) can be substituted for $k_{r}$. The spacing between adjacent CTFs now simplifies to

$$
\Delta k_{\theta}=2 k_{\rho}\left[\sin \frac{\Delta \theta}{2}+\frac{\lambda}{2}\left(k_{\rho}-\frac{2 \alpha}{\lambda}\right) \cos \frac{\Delta \theta}{2}\right] .
$$

The maximum measurable object size is inversely related to the distance between CTFs as $D=1 / \Delta k_{\theta}$. We can use Eq. (A2) to define the maximum size, $D$, of a reconstruction at a given convergence semiangle, electron wavelength, tilt step, and maximum spatial frequency.

$$
D=\frac{1}{\lambda k_{\rho}{ }^{2}\left[\cos \frac{\Delta \theta}{2}+\frac{1}{\lambda k_{\rho}}\left(2 \sin \frac{\Delta \theta}{2}-2 \alpha \cos \frac{\Delta \theta}{2}\right)\right]} .
$$

Introducing $d=1 / k_{\rho}$ to relate maximum spatial frequency to resolution, we change Eq. (A3) to

$$
D=\frac{d^{2}}{\lambda\left[\cos \frac{\Delta \theta}{2}+\frac{d}{\lambda}\left(2 \sin \frac{\Delta \theta}{2}-2 \alpha \cos \frac{\Delta \theta}{2}\right)\right]} .
$$

Complete information transfer occurs when adjacent CTFs overlap. The distance between adjacent CTFs is zero at this point (labeled $k_{c}$ in Fig. 2), so we set Eq. (A2) to zero to find $k_{c}$.

$$
k_{c}=\frac{2}{\lambda}\left(\alpha-\tan \frac{\Delta \theta}{2}\right) .
$$

Under the small angle approximation $\tan (\Delta \theta / 2) \approx \Delta \theta / 2$,

$$
k_{c}=\frac{2 \alpha-\Delta \theta}{\lambda} .
$$

Equations (A3) and (A4) are only valid for $k_{c} \leq k_{\rho} \leq$ $k_{\max }$ or $1 / k_{\max } \leq d \leq 1 / k_{c}$. For $0 \leq k_{\rho} \leq k_{c}$ and $1 / k_{c} \leq d$, complete information is collected and the maximum object size is unbounded, giving piecewise equations. Using firstorder small angle approximations $[\sin (\Delta \theta / 2) \approx \Delta \theta / 2$ and $\cos (\Delta \theta / 2) \approx 1]$, Eq. (A5), and piecewise definition of maximum object size, Eqs. (A3) and (A4) become, respectively,

$$
\begin{gathered}
D= \begin{cases}\infty, & 0 \leq k_{\rho} \leq k_{c} \\
\frac{1}{\lambda k_{\rho}^{2}\left(1-\frac{k_{c}}{k_{\rho}}\right)}, & k_{c}<k_{\rho} \leq \frac{2 \alpha}{\lambda}\end{cases} \\
D= \begin{cases}\infty, & \infty>d \geq \frac{1}{k_{c}} \\
\frac{d^{2}}{\lambda\left(1-k_{c} d\right)}, & \frac{1}{k_{c}}>d \geq \frac{\lambda}{\alpha}\end{cases}
\end{gathered}
$$

Equations (A6) and (A7) define the relationship between object size and resolution for aberration-corrected tomography analogous to the Crowther limit.

\section{APPENDIX B: SAMPLING FROM A SINGLE FOCAL PLANE}

To understand how information is sampled using defocus, consider a single projection taken at focal plane, $\Delta z$. Under an incoherent linear imaging model an image, $\mathcal{I}$, at defocus $\Delta z$ is a slice of the convolution of the object, $\mathcal{O}(\mathbf{r})$, with the electron probe, $h(\mathbf{r})$ :

$$
\begin{aligned}
& \mathcal{I}(\mathbf{r})=[\mathcal{O}(\mathbf{r}) \otimes h(\mathbf{r})] \quad \delta(z-\Delta z) \\
& =[\bigcirc \otimes \mathbf{X}] \Delta \Delta \\
& \mathcal{I}(\mathbf{k})=\left[\begin{array}{ll}
\mathcal{O}(\mathbf{k}) & h(\mathbf{k})
\end{array}\right] \quad \otimes e^{-i k_{z} \Delta z} \delta\left(k_{x}\right) \delta\left(k_{y}\right) \\
& =\left[\begin{array}{ll}
\otimes & \infty
\end{array}\right] \otimes
\end{aligned}
$$

In $k$ space, this is a multiplication followed by convolution with a rod whose phase oscillates according to defocus Eq. (B2). Evaluating the convolution in Eq. (B2) and rearranging, we find

$$
\mathcal{I}\left(k_{x}, k_{y}, \Delta z\right)=\int_{-\infty}^{\infty} d k_{z}^{\prime} \mathcal{O}\left(k_{x}, k_{y}, k_{z}^{\prime}\right) h\left(k_{x}, k_{y}, k_{z}^{\prime}\right) e^{-i \Delta z k_{z}^{\prime}}
$$

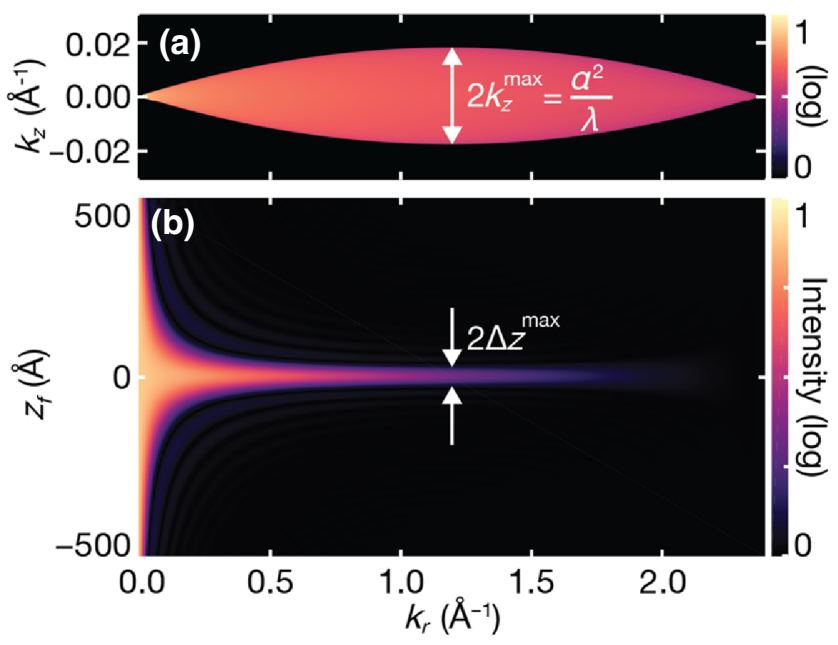

FIG. 6. (a) CTF of a through-focal image stack and the (b) CTF Fourier transform along $k_{z}$ for a $200-\mathrm{keV}, 30-\mathrm{mrad}$ aberration-free electron beam. This illustrates that the midband frequencies set the maximum defocus step required for sampling with defocus. When the beam is out of focus from a specimen feature only low frequencies are transferred. Surprisingly, the highest frequencies are also transferred but the information intensity is too low to be useful. 
This is a Fourier transform in only one dimension from $k_{z}$ space to $\Delta z$ space. Figure 6 shows this function for an aberration-free beam and a point object - it illustrates how information in the midband is only measured within a limited focal range $\left(2 \Delta z^{\max }\right)$. Nyquist sampling sets the depth resolution at $\Delta z^{\max }=1 / k_{z}^{\max }=\lambda / \alpha^{2}$, which defines the depth of focus - derived herein using wave optics.

\section{APPENDIX C: POISSONIAN-GAUSSIAN NOISE MODELING OF (S)TEM IMAGES}

An experimentally measured noisy (S)TEM image, $\widetilde{p}(x, y)$, of a projected specimen, $p(x, y)$, is adequately modeled with both Poisson and Gaussian noise. With dose rate $\chi$, acquisition time $t$, a noisy image becomes

$$
\tilde{p}(x, y)=\chi t p(x, y)+\chi t n_{p}[0, \chi t p(x, y)]+t n_{g}\left(0, \frac{\sigma^{2}}{t}\right) .
$$

The first two terms describe Poisson statistics and the last term follows Gaussian statistics [24]. The Poisson noise is a function of both the specimen and dose $(\chi t)$ and the noise term $n_{p}$ is mean centered. The Gaussian noise $n_{g}$ is specimen independent, dose independent, mean-centered, and has variance $\sigma^{2} / t$ described by the central limit theorem. The measured image is the expected value of our measurement, $E[\widetilde{p}(x, y)]=\chi t p(x, y)$, and noise adds signal variance, $\operatorname{Var}[\widetilde{p}(x, y)]=\chi t p(x, y)+t \sigma^{2}$. The variance has two terms from the Gaussian and Poisson noise.

The SNR of a (S)TEM image is

$$
\begin{aligned}
\mathcal{R}[\tilde{p}(x, y)] & =\frac{E\left[\widetilde{p}^{2}(x, y)\right]}{\operatorname{Var}[\widetilde{p}(x, y)]}=1+\frac{\{E[\widetilde{p}(x, y)]\}^{2}}{\operatorname{Var}[\widetilde{p}(x, y)]} \\
& =1+\frac{\chi^{2} t^{2} p^{2}(x, y)}{\chi t p(x, y)+t \sigma^{2}} .
\end{aligned}
$$

A (S)TEM image is Poisson limited $\left[\chi t p(x, y) \gg t \sigma^{2}\right]$ for large signals or high detector efficiency and the SNR depends only on the object and total dose.

$$
\mathcal{R}[\tilde{p}(x, y)]=1+\chi t p(x, y) .
$$

\section{APPENDIX D: DOSE FRACTIONATION FOR THROUGH-FOCAL IMAGING}

Here we show the SNR of useful information in a through-focal stack of images is only dependent on the total dose, so long as the through-focal stack is oversampled along defoci. Each image is taken at a beam defocus value $\left(z_{f}\right)$ and, for a through-focal acquisition oversampled by a factor of $M\left(M \Delta z<\lambda / \alpha^{2}\right), M$ adjacent defoci can be summed without any loss of information. $\delta z$ is the defocus step size. The SNR after summing $M$ adjacent defoci, each with dose per image $\chi t$ at defoci $z_{f}+m \delta z$, describes the quality of useful information. Because each acquired image is independently measured the expected value and variance of the sum adds linearly: $E\left[\sum_{m} \tilde{p}\left(x, y, z_{f}+m \Delta z\right)\right]=$ $M \chi t \quad \bar{p}\left(x, y, z_{f}\right), \quad \operatorname{Var}\left[\sum_{m} \tilde{p}\left(x, y, z_{f}+m \Delta z\right)\right]=M \chi t \bar{p}$ $\left(x, y, z_{f}\right)+M t \sigma^{2} \cdot \bar{p}\left(x, y, z_{f}\right)$ denotes the averaged image. Therefore, SNR after summing adjacent defocused images is

$$
\begin{gathered}
\mathcal{R}\left[\sum_{m} \widetilde{p}\left(x, y, z_{f}+m \Delta z\right)\right]= \\
1+\frac{\left[M \chi t \bar{p}\left(x, y, z_{f}\right)\right]^{2}}{M \chi t \bar{p}\left(x, y, z_{f}\right)+M t \sigma^{2}} .
\end{gathered}
$$

For Poisson-noise-limited images the SNR becomes

$$
\mathcal{R}\left[\sum_{m} \tilde{p}\left(x, y, z_{f}+m \Delta z\right)\right]=1+M \chi t \bar{p}\left(x, y, z_{f}\right) .
$$

Therefore, the SNR of an oversampled through-focal stack only depends on the total dose $(M \times t)$.

[1] A. Klug and R. A. Crowther, Three-dimensional image reconstruction from the viewpoint of information theory, Nature 238, 435 (1972).

[2] R. Hegerl and W. Hoppe, Influence of electron noise on three-dimensional image reconstruction, Z. Naturforsch. A 31, 1717 (1976).

[3] D. J. De Rosier and A. Klug, Reconstruction of three dimensional structures from electron micrographs, Nature 217, 130 (1968).

[4] P. A. Midgley, M. Weyland, J. M. Thomas, and B. F. G. Johnson, Z-contrast tomography: A technique in threedimensional nanostructural analysis based on Rutherford scattering, Chem. Commun. 10, 907 (2001).

[5] M. C. Scott, C.-C. Chen, M. Mecklenburg, C. Zhu, R. Xu, P. Ercius, U. Dahmen, B. C. Regan, and J. Miao, Electron tomography at 2.4-ångström resolution, Nature 483, 444 (2012).

[6] Y. Yang, C.-C. Chen, M. C. Scott, C. Ophus, R. Xu, A. Pryor, L. Wu, F. Sun, W. Theis, J. Zhou, M. Eisenbach, P. R. C. Kent, R. F. Sabirianov, H. Zeng, P. Ercius, and J. Miao, Deciphering chemical order/disorder and material properties at the single-atom level, Nature 542, 75 (2017).

[7] S. Van Aert, K. J. Batenburg, M. D. Rossell, R. Erni, and G. Van Tendeloo, Three-dimensional atomic imaging of crystalline nanoparticles, Nature 470, 374 (2011).

[8] O. L. Krivanek, N. Delby, and A. R. Lupini, Towards sub- $\AA$ electron beams, Ultramicroscopy 78, 1 (1999).

[9] P. E. Batson, N. Delby, and O. L. Krivanek, Subångstrom resolution using aberration corrected electron optics, Nature 418, 617 (2002).

[10] P. D. Nellist, M. F. Chisholm, N. Dellby, O. L. Krivanek, M. F. Murfitt, Z. S. Szilagyi, A. R. Lupini, A. Borisevich, 
W. H. Sides Jr., and S. J. Pennycook, Direct sub-angstrom imaging of a crystal lattice, Science 305, 1741 (2004).

[11] D. A. Muller, L. F. Kourkoutis, M. Murfit, J. H. Song, H. Y. Hwang, J. Silcox, N. Dellby, and O. L. Krivanek, Atomic-scale chemical imaging of composition and bonding by aberration-corrected microscopy, Science 319, 1073 (2008).

[12] G. Behan, E. C. Cosgriff, A. I. Kirkland, and P. D. Nellist, Three-dimensional imaging by optical sectioning in the aberration-corrected scanning transmission electron microscope, Philos. Trans. R. Soc. A-Math. Phys. Eng. Sci. 367, 3825 (2009).

[13] R. Hovden, H. L. Xin, and D. A. Muller, Extended depth of field for high-resolution scanning transmission electron microscopy, Microsc. Microanal. 17, 75 (2011).

[14] H. Yang, J. G. Lozano, T. J. Pennycook, L. Jones, P. B. Hirsch, and P. D. Nellist, Imaging screw dislocations at atomic resolution by aberration-corrected electron optical sectioning, Nat. Commun. 6, 7266 (2015).

[15] K. van Benthem, A. R. Lupini, M. Kim, H. S. Baik, S. Doh, J.-H. Lee, M. P. Oxley, S. D. Findlay, L. J. Allen, J. T. Luck, and S. J. Pennycook, Three-dimensional imaging of individual hafnium atoms inside a semiconductor device, Appl. Phys. Lett. 87, 034104 (2005).

[16] R. Hovden, P. Ercius, Y. Jiang, D. Wang, Y. Yu, H. D. Abruña, V. Elser, and D. A. Muller, Breaking the crowther limit: Combining depth-sectioning and tilt tomography for high-resolution, wide-field $3 \mathrm{~d}$ reconstructions, Ultramicroscopy 140, 26 (2014).

[17] T. Dahmen, J.-P. Baudoin, A. R. Lupini, C. Kübel, P. Slusallek, and N. de Jonge, Combined scanning transmission electron microscopy tilt- and focal series, Microsc. Microanal. 20, 548 (2014).

[18] B. E. H. Saxberg and W. O. Saxton, Quantum noise in 2D projections and 3D reconstructions, Ultramicroscopy $\mathbf{6}, 85$ (1981).

[19] R. A. Crowther, D. J. DeRosier, and A. Klug, The reconstruction of a three-dimensional structure from projections and its application to electron microscopy, Proc. R. Soc. London, Ser. A 317, 319 (1970).

[20] R. N. Bracewell and A. C. Riddle, Inversion of fanbeam scans in radio astronomy, Astrophys. J. 150, 427 (1967).

[21] R. N. Bracewell, Numerical transforms, Science 248, 697 (1990).

[22] See Supplemental Material at http://link.aps.org/supple mental/10.1103/PhysRevApplied.15.014003 for additional details on the convergent beam CTF, multislice simulated aberration-corrected tomography reconstruction, and figures comparing information collection in traditional and aberration-corrected tomography.

[23] V. Intaraprasonk, H. L. Xin, and D. A. Muller, Analytic derivation of optimal imaging conditions for incoherent imaging in aberration-corrected electron microscopes, Ultramicroscopy 108, 1454 (2008).

[24] A. Foi, M. Trimeche, V. Katkovnik, and K. Egiazarian, Practical poissonian-gaussian noise modeling and fitting for single-image raw-data, IEEE Trans. Image Process. 17, 1737 (2008).

[25] B. F. McEwen, M. Marko, C.-E. Hsieh, and C. Mannella, Use of frozen-hydrated axonemes to assess imaging parameters and resolution limits in cryoelectron tomography, J. Struct. Biol. 138, 47 (2002).

[26] E. J. Kirkland, Advanced Computing in Electron Microscopy (Springer, US, 2010), 2nd ed.

[27] A. Jeffrey and D. Zwillinger, Table of Integrals, Series, and Products (Elsevier, Amsterdam, 2007), 8th ed.

[28] A. Pryor Jr., C. Ophus, and J. Miao, A streaming multi-GPU implementation of image simulation algorithms for scanning transmission electron microscopy, Adv. Struct. Chem. Imaging 3, 15 (2017).

[29] Y. Yang, C. C-C M. C. Scott, C. Ophus, R. Xu, A. Pryor, L. Wu, F. Sun, W. Theis, J. Zhou, M. Eisenbach, P. R. C. Kent, R. F. Sabirianov, P. Zeng, H. Ercius, and J. Miao, 3D chemical order/disorder of an fept nanoparticle, Materials Data Bank FePt00001. 